\title{
Implementation of Interactive Web 3D Based Virtual Educational System using O3D-WebGL
}

\author{
Kwang-Ho Seok ${ }^{1}$ and Yoon Sang $\mathrm{Kim}^{1}$ \\ ${ }^{1}$ Department of Computer Science and Engineering \\ yoonsang@koreatech.ac.kr
}

\begin{abstract}
This paper proposes an interactive Web $3 D$ based virtual educational s stem using $O 3 D$ WebGL. The proposed system is capable of providing flexible services without being confined to a specific platform. A virtual educational content was implemented with an educational goal of "saving the earth from global warming." The developed virmav educational content of global warming consists of two subjects: Ama on and Arctic. The effectiveness of the proposed virtual education system was verified based on implementation of user interaction. A storytelling approach was employed to maximize the interactive experience, and the $3 D$ environment was implemented using $O 3 D^{\circ}$ of WebGL to provide a user-oriented interface, as well as low-capacity, high-quality data sentices.
\end{abstract}

Keywords: Web 3D, virtual educational content. $3 D-W e b G L$

\section{Introduction}

As the Internet becomes popular with various types of content flooding the Web, there is an increasing need for securing high quality Web contents. Web based interactive virtual educational enyironment is an mportant method that can be used to replace the off-line education. Rapid technologieal advances are prompting Web contents to make a transition from 2D to 3D in order to enhance satisfaction for the users [1-5]. Recently, research works on Web 3D based e-learning system for healthcare education have also been extensively conducted [6-8]. There are also active research and development activities regarding experience halls and exhibition spaces using virtual reality [9-13]. It is difficult to satisfy visitors at vitúal educational contents that mostly use static texts and images. From the development perspective, there is a need to make improvements on the conventional method of creating contents that are difficult to add and modify, and develop reusable Web 3D contents that are easy to update and renew.

This paper proposes an interactive Web 3D based virtual educational system using O3DWebGL. The proposed system is capable of providing flexible services without being confined to a specific platform. A virtual educational content was implemented with an educational goal of "saving the earth from global warming." The developed virtual educational content of global warming consists of two subjects such as "Amazon" and "Arctic". The effectiveness of the proposed virtual educational system was verified based on implementation of user interaction. A storytelling approach was employed to maximize the interactive experience, and the 3D environment was implemented using O3D of WebGL to provide a user-oriented interface, as well as low-capacity, high-quality data services. By applying a next-generation Web 3D technology, we were able to meet various demands from users and enhance the level of satisfaction. 


\section{Design of the Web 3D-Based Virtual Educational Platform using O3D- WebGL}

In this section, we present the design of the Web 3D based virtual educational platform using O3D-WebGL. Competition is becoming fierce in the global web browser and OS markets. Since the initial development of VRML, there have been active development and research activities in the web 3D technology for implementing 3D graphic on the Web browser. WebGL [14] is a new standard for 3D drawing on the Web. By providing hardware 3D rendering for Canvas in HTML5, WebGL allows system graphics card to render 3D scenes and models in browsers. Amidst developments of various Web 3D technologies and software solutions, Google recently introduced O3D [15], an open-source Web ADI for implementing 3D graphic on Web browsers such as the Chrome. Unlike other Web 3D environments, O3D is easy to install because it uses a Web plug-in API based on WebGL, and there is no need for additional programs. Furthermore, Q3D runs on various operating systems, including Windows, Mac and Linux, and is compatible with popular browsers, such as Google Chrome, Mozilla Firefox, Microsoft Internet Explorer and Apple Safari. Recently, the WebGL implementation of O3D works in any WebGL-enabled browser. In this paper, a Web 3D based virtual educational system is designed using O3D-WebGL. The proposed interactive web 3D based virtual educational system is capable of providing flexible services without being confined to a specific platform. In particular, the interactive Web 3D based virtual educational system proposed in this paper is based on the HTML Web standard, so users that access the Internet through different means can receive the information without being alienated. The proposed $\mathrm{NeB} B \mathrm{D}$ virtuat environment is based on a hierarchical architecture, which facilitates expansion of various forms of Web content and removal of existing data. This paper explains the development of the virtual environment, which has an educational objective of "saving the earth from global warming." Figure 1 show the expandable hierarchicalarchitecture of the proposed virtual educational platform.

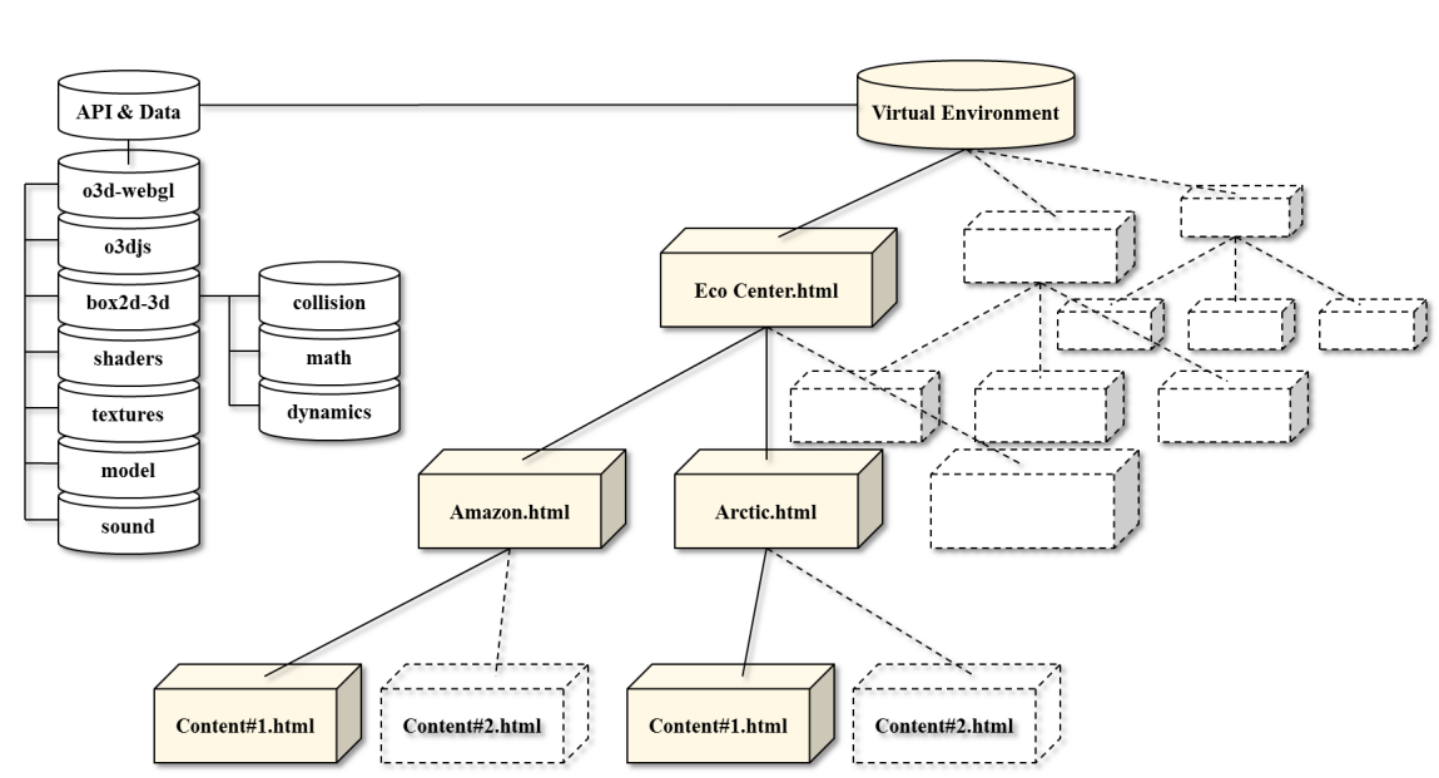

Figure 1. Expandable hierarchical architecture of the proposed virtual educational platform 
The classes of the proposed virtual educational platform are included in the WebGL implementation of O3D. The virtual environments are composed of various 3D Web environments as follwings: Eco Center, Amazon, Arctic, Contents of Amazon, and Contents of Arctic. Participants can access a wide range of educational materials through user-oriented multimedia data, including texts, sounds, and images at the various virtual environments.

User-oriented storytelling is regarded as one of the most important principles in designing educational materials because it enables interactive education, allowing students to become emersed in learning with a sense of reality. Adding game elements to the storytelling approach further enhances education by offering enjoyable and interesting learning experience. The Web 3D virtual environment proposed in this paper provides user-oriented experience. In addition to accomplishing missions in the $3 \mathrm{D}$ virtual environment participants can experience various places of their choice.

As shown in Figure 2, the interactive Web 3D virtual educational contents based on storytelling consists of the Eco Center, Amazon Space, and A Actic Space. Each environment provides user-oriented storytelling. Participants can receive quality/education from informative stories in various environments and a wide range of multimedia. Participants can choose amazon space or arctic space at the Eco Center. As participants venture into different places of the virtual environment, they will find red eco gauges, Indicating that there is a mission to be accomplished. Once the mission is complete, eco gauge turns green. Participants can learn about the cause of global warming (Rogging, Slash-and-burn) at the Amazon. As well, Learning Center in the Amazon prowes various educational data. Melting glaciers is an example of the effects of global warming, Furthermore, the polar bear is another example of the effects of global warming. These are the effects by global warming which can be experienced at the Arctic. Also, Learning Center in the Arctic provides various educational data.

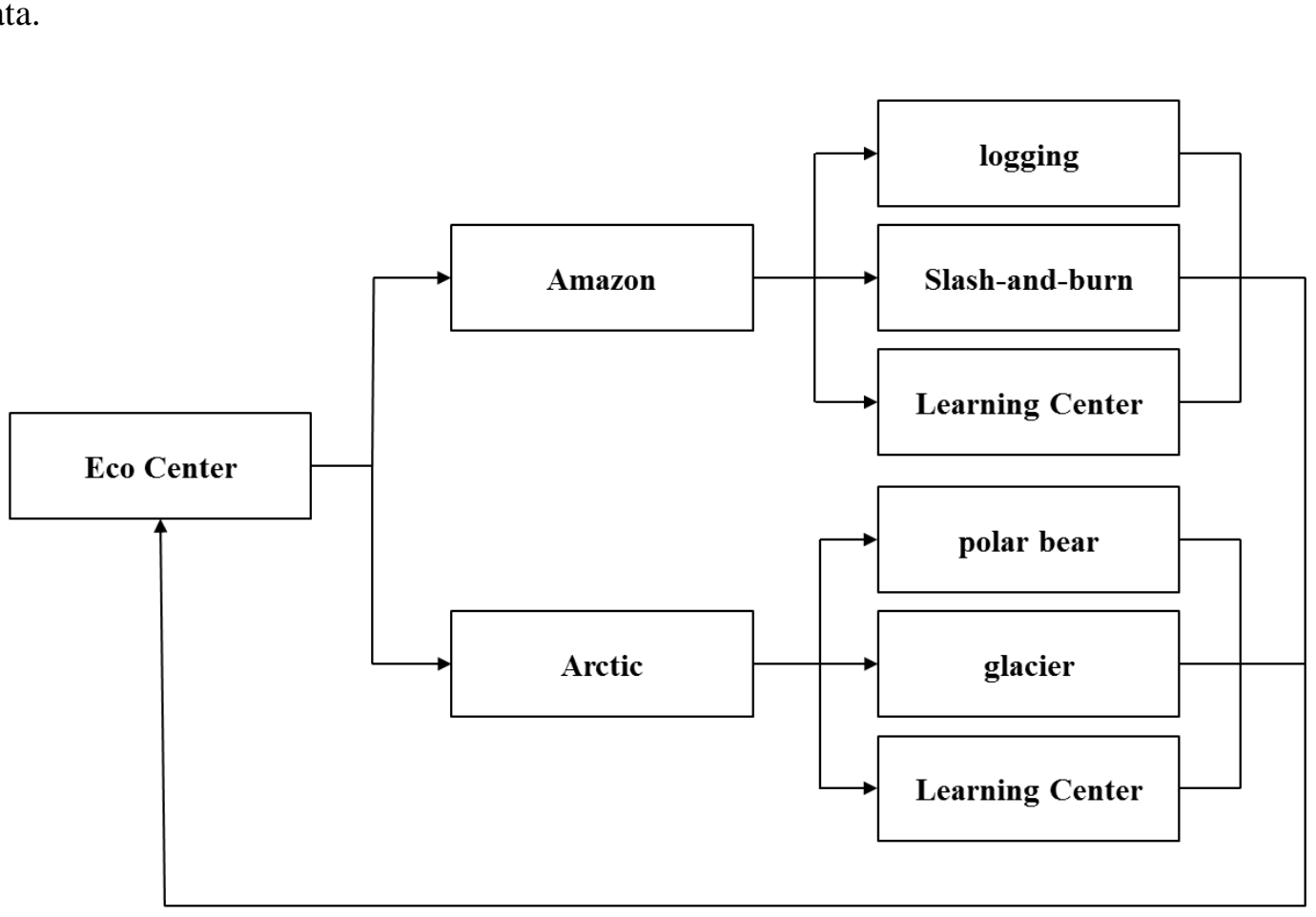

Figure 2. Interactive Web 3D based virtual educational contents structure 


\section{Implementation of the Interactive Virtual Education Contents}

\subsection{D Modeling of Virtual Environment}

The interactive 3D virtual environment developed by this study based on O3D uses a storytelling approach to maximize the interactive experience. Moreover, the $3 \mathrm{D}$ environment was implemented using O3D to provide a user-oriented interface, as well as low-capacity, high-quality data services.

O3D is a very powerful open-source Web API for developing interactive 3D applications for the Web browser. In this paper, O3D was used to implement the 3D virtual experience environment on the Web browser and to maximize the capability of providing interactive experience. The 3D virtual environment and its objects were modeled using the 3D MAX tool, and interactive experience functions were programmed in Java script using Q3D. Figure 3 shows the maps modeled in 3D. The developed 3D environments proyide three maps such as the Eco Center, Amazon, and Arctic.

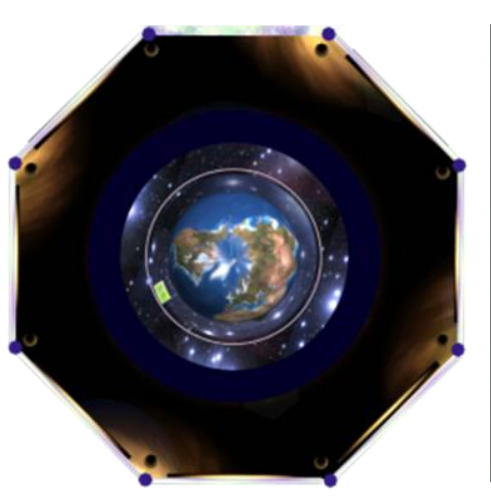

(a) Eco Center

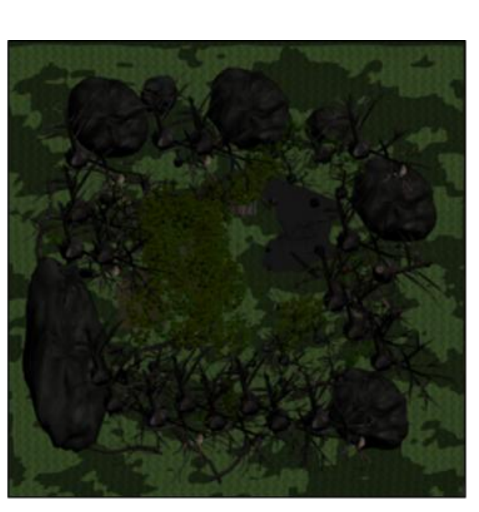

(b) Amazon

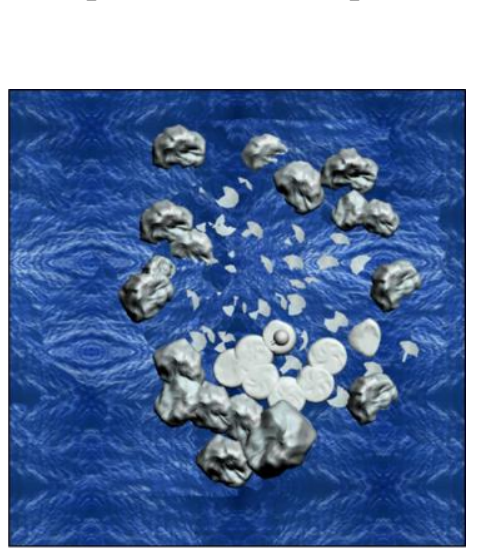

(C) Arctic Figure 3. The modeled maps in 3D (top view)

Figure 4 shows the virtaa $3 \mathrm{D}$ environment of the Eco Center. The hall of Eco Center was modeled as hexagonal structure. Also, a large terrestrial globe is located in the center of the hall.

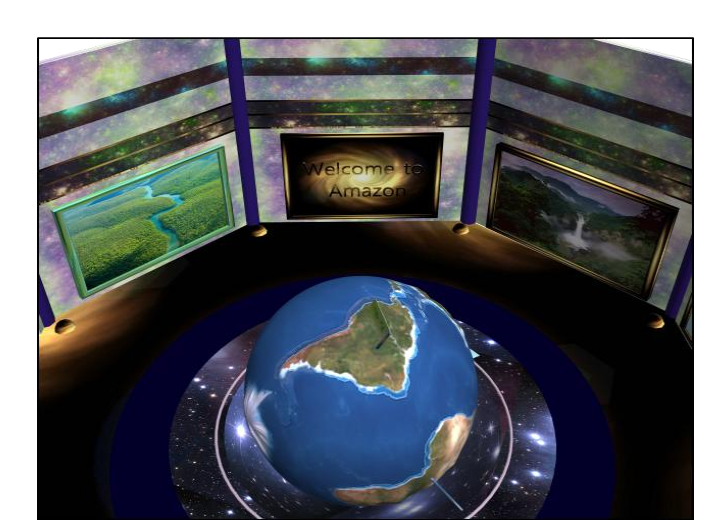

(a) Amazon view in the Eco Center

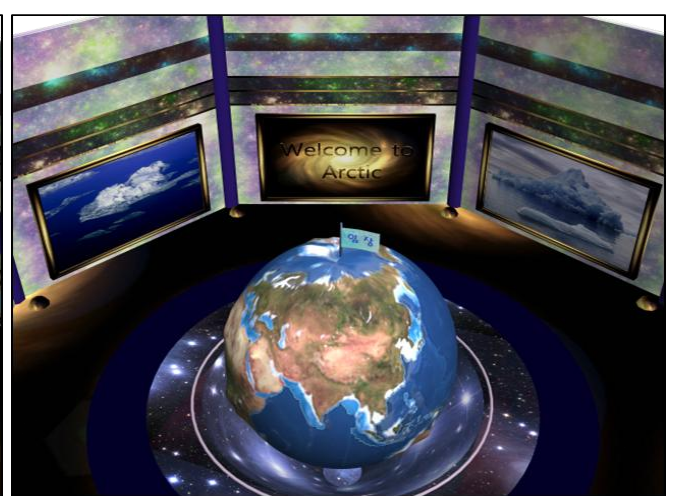

(b) Arctic view of in the Eco Center

Figure 4. Virtual 3D environment of the Eco Center 
Figure 5 shows the virtual 3D environments of the Amazon and Arctic. Amazon and the Arctic were modeled as a rectangular structure. Each terrain contains a Learning Center. The Learning Center of the Amazon was modeled as a log house. In addition, the Learning Center of the Arctic was modeled as an igloo.

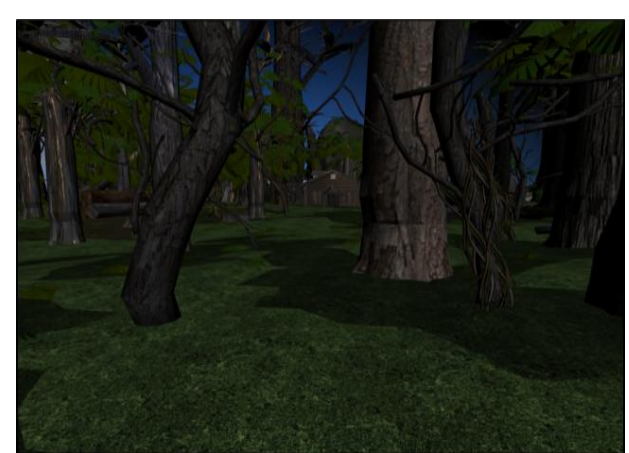

(a) Terrain of the Amazon

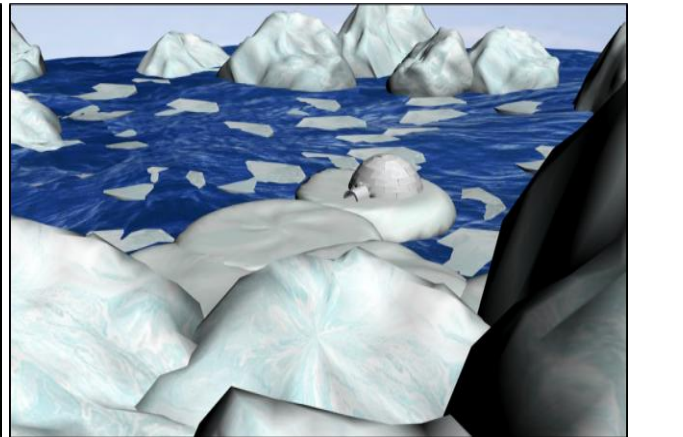

(b) Terrain of the Arctic

Figure 5. Virtual 3D environment of Amazon and Arctic

\subsection{Implementation of Interactive Web $3 \mathrm{D}$ Based Virtual Educational Contents}

Participants of the Web 3D virtual epvironment firsfenter the Eco Center. The user view is constructed from the first-personperspective. At the,Eco Center, participants can move the earth object in the center with the mouse and click a point to go to a desired location between the Amazon and the Arctic. The Web 3D viftualenvironment implemented in this study has a very strong capability for expanding thecontent. The developer can easily add a new location alongside the Amazon and the Arctic and provide related contents. Participants can access a wide range of educational materials through user-oriented multimedia data, including texts, sounds, and inages at the Eco Center. Figure 6 shows the Interactive Web 3D based contents of the Eco Center.

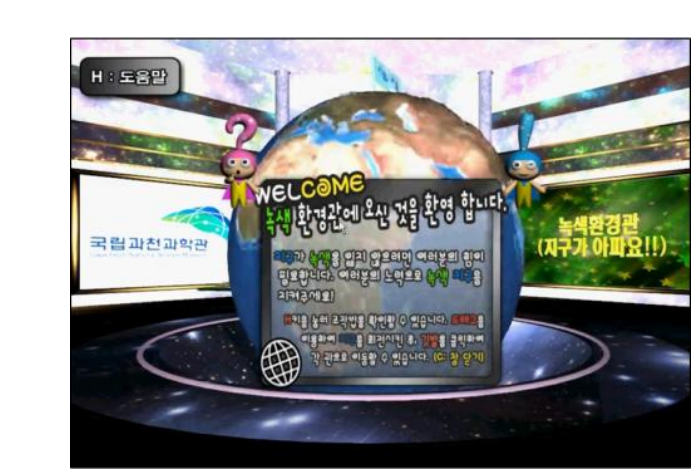

(a) Educational environment \#1

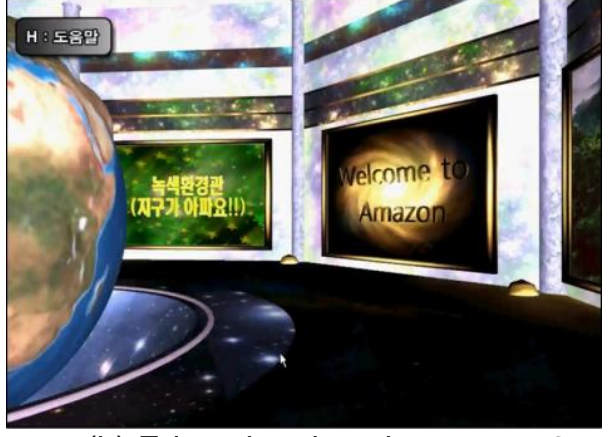

(b) Educational environment \#2 


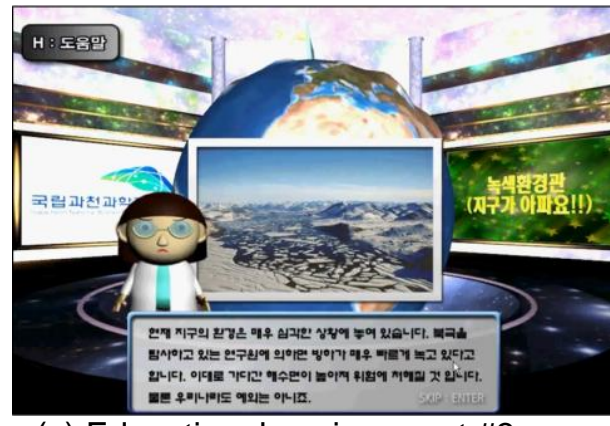

(c) Educational environment \#3

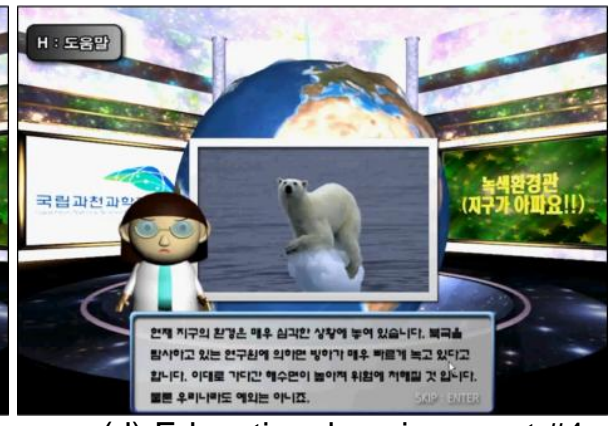

(d) Educational environment \#4

Figure 6. Interactive Web 3D based contents of the Eco Center

A participant can move the earth object at the center of the Eco Center and go to the Amazon region with a mouse click. At the Amazon, the participant is able to explore various places using the keyboard. Participants can learn about the cause of global warming (logging, Slash-and-burn) at the Amazon. During the exploration, participants will find red eco gauges, indicating that there is a mission to be, accomplished. Once the mission is complete, eco gauge turns green. Figure 7 shows interactive Web 3D based contents of the Amazon.

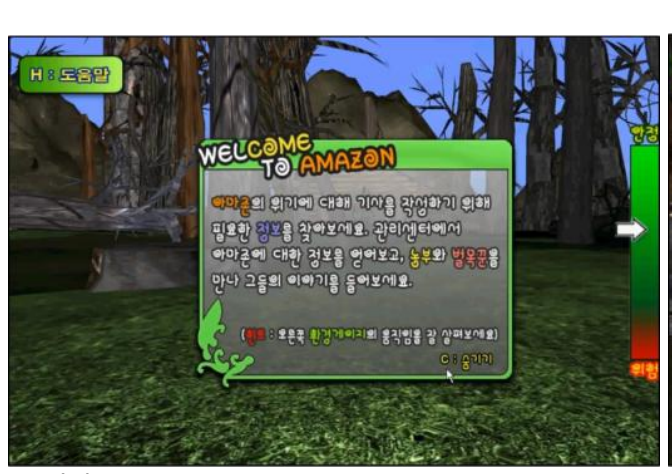

(a) Educational environment \#1 Or

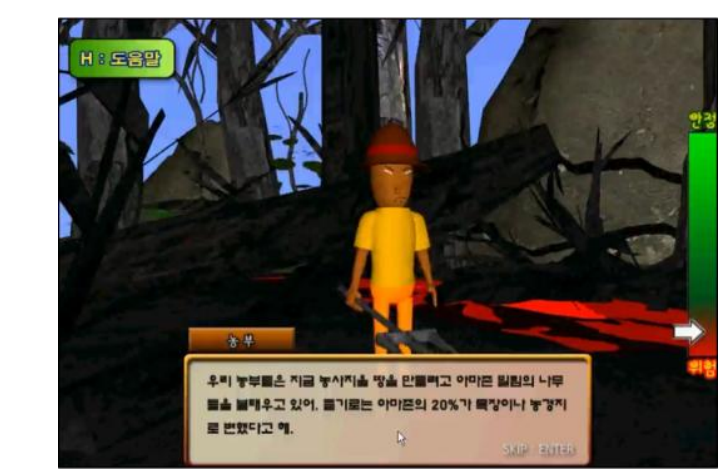

(c) Educational environment \#3

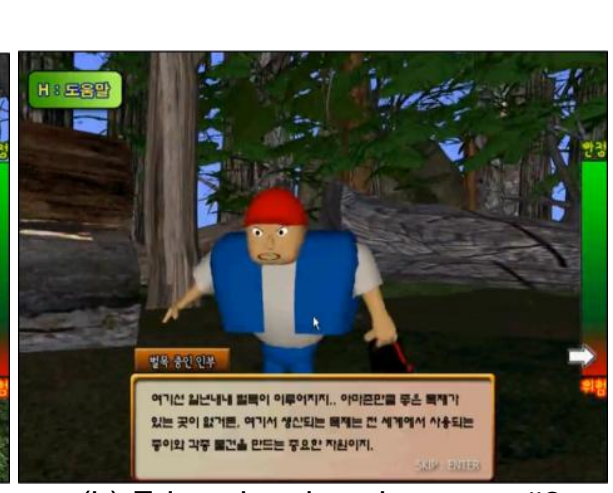

(b) Educational environment \#2

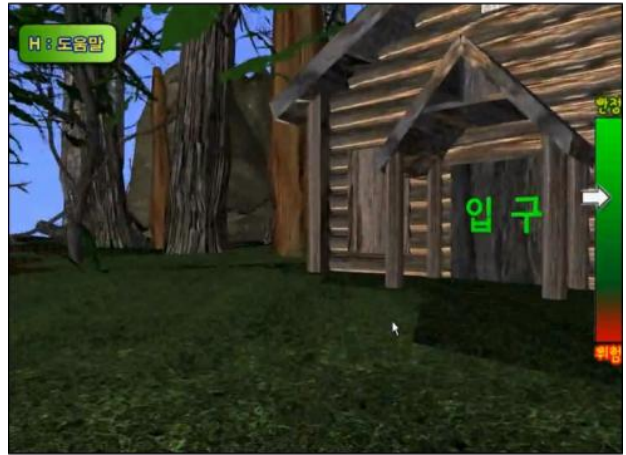

(d) Educational environment \#4

Figure 7. Interactive Web 3D based contents of the Amazon

Figure 8 shows the educational contents of learning center in the Amazon. Amazon's Learning Center is modeled as a log house. A participant can accomplish all of the missions in the Amazon region by entering the Learning Center. Learning Center 
provides six kinds of educational contents. Those educational contents are controlled using either mouse clicks or keyboard inputs.

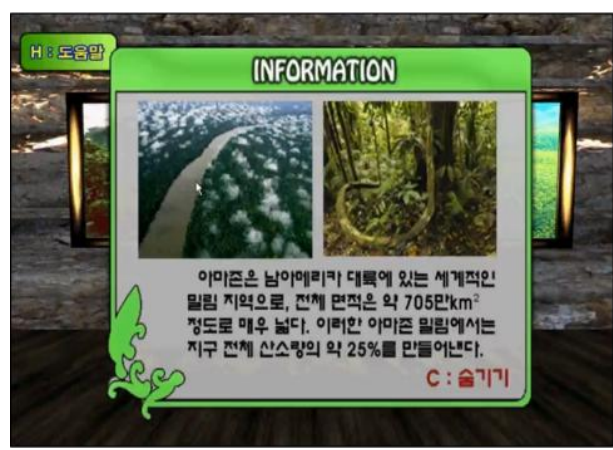

(a) Educational Content \#1

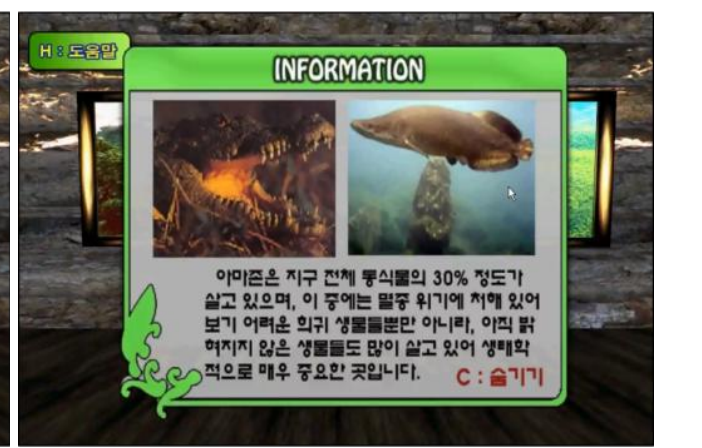

(b) Educational Content \#2

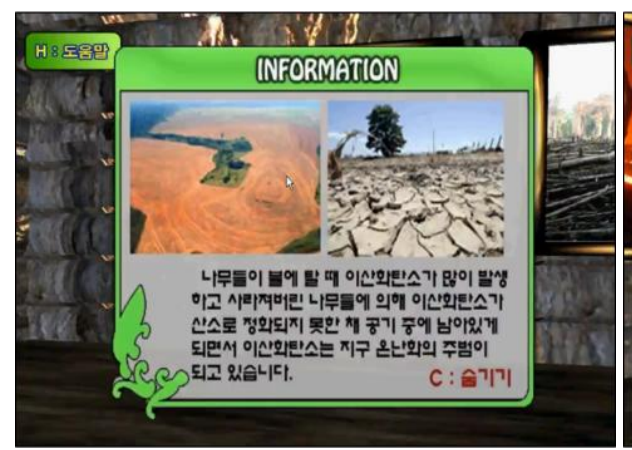

(c) Educational Content \#3

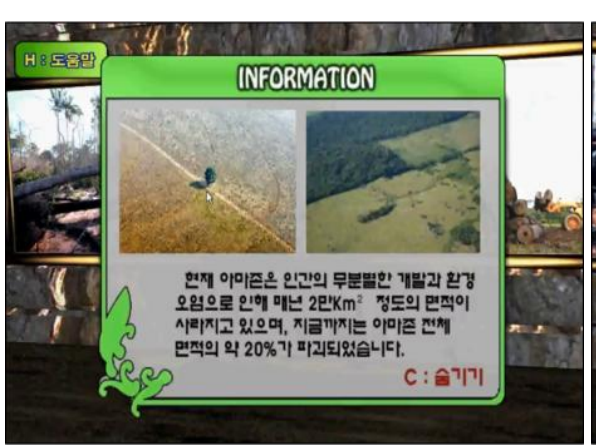

(e) Educational Content \#5

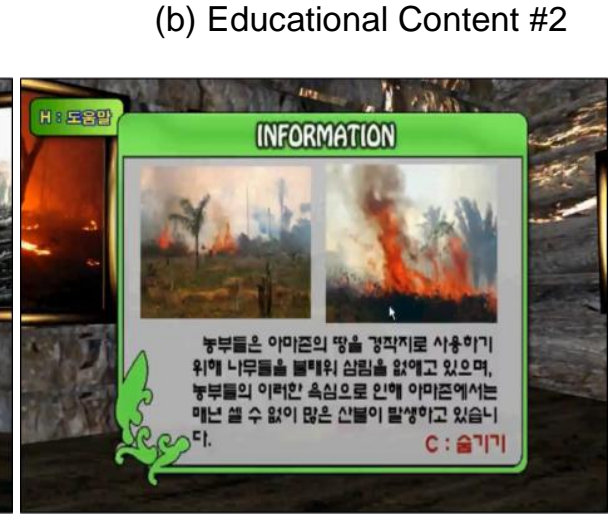

(d) Educational Content \#4

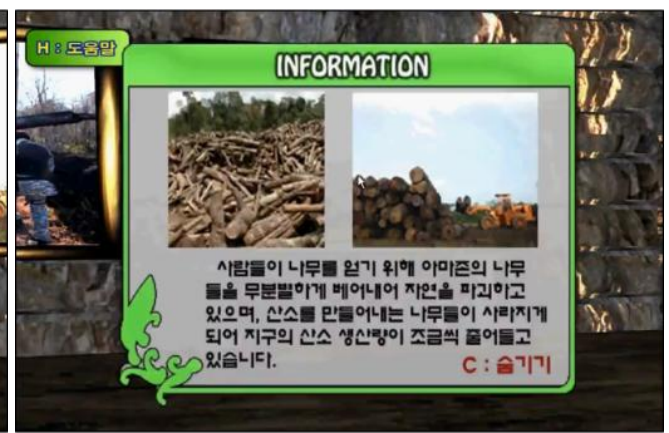

(f) Educational Content \#6

Figure 8. Educational contents of Learning Center in the Amazon

A participant can move the earth object at the center of the Eco Center and go to the Arctic region with a mouse click. The Arctic region also offers missions similar to those in the Amazon. At the Arctic, the participant is able to explore various places using the keyboard. Adding game elements to the storytelling approach further enhances education by offering enjoyable and interesting learning experience. Melting glaciers is an example of the effects on global warming. Furthermore, the polar bear is another example of the effects on global warming. These are the effects on global warming which can be experienced at the Arctic. During the exploration, participants will find 
red eco gauges, indicating that there is a mission to be accomplished. Once the mission is complete, eco gauge turns green. Furthermore, participants can engage in various interactions and access educational materials through multimedia information regarding the Arctic region. Figure 9 shows interactive Web 3D based contents of the Arctic.

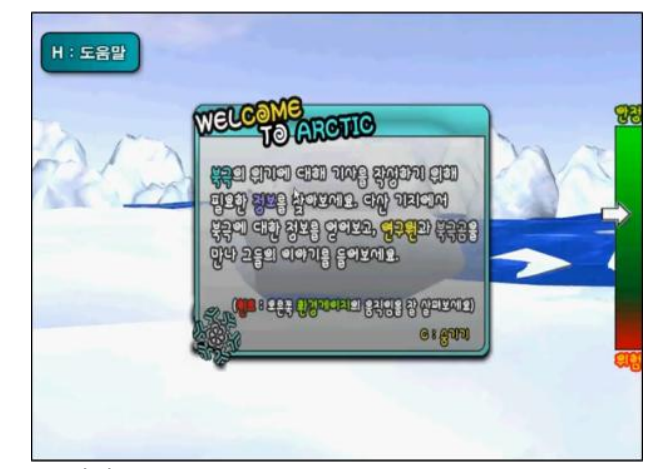

(a) Educational environment \#1

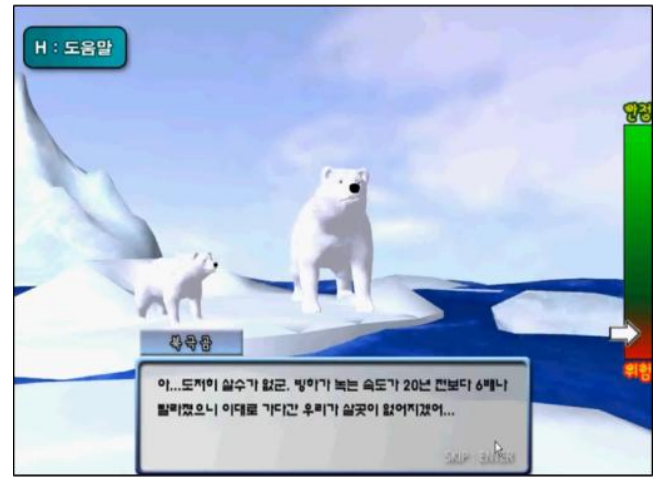

(c) Educational environment \#3

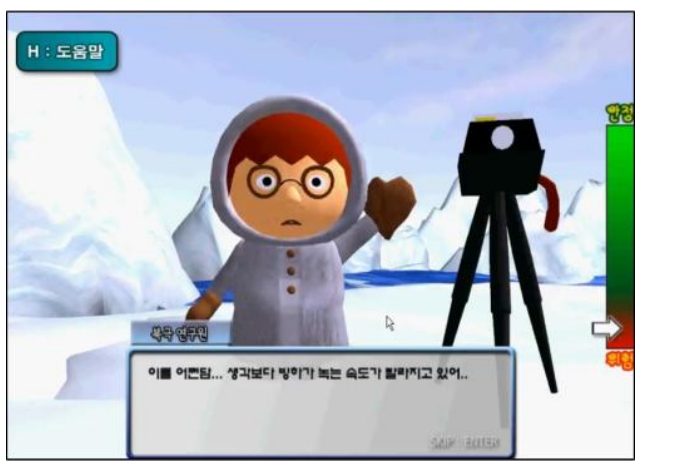

(b) Educational environment \#2

Figure 9. Interadtive Web 3D based contents of the Arctic

Figure 10 shows the educational contents of Learning Center in the Arctic. A participant can acomplish all of the missions in the Arctic region by entering the Learning Center. Learning Center provides six kinds of educational contents. Those educational contents are controlled using either mouse clicks or keyboard inputs.

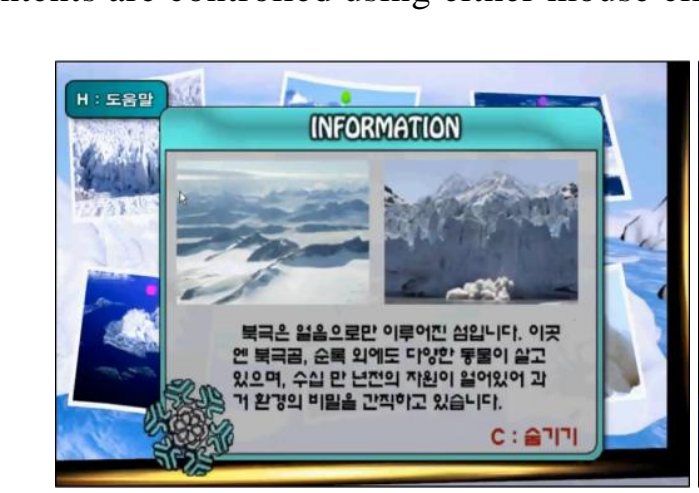

(a) Educational Content \#1

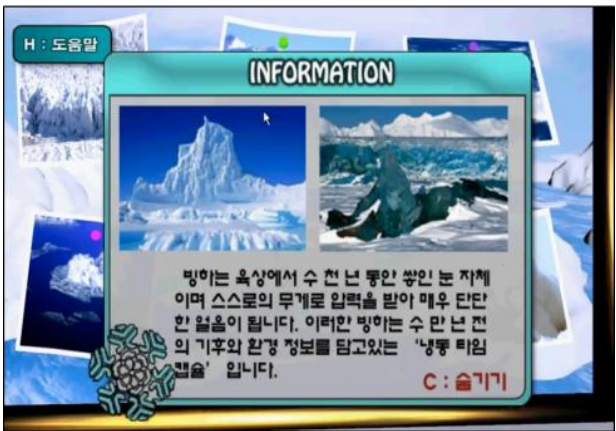

(b) Educational Content \#2 


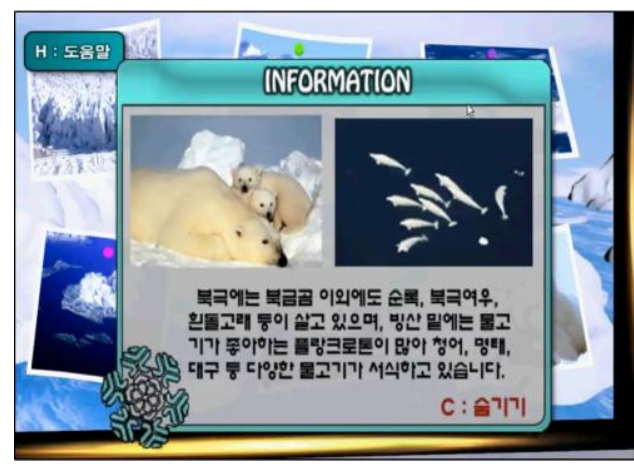

(c) Educational Content \#3

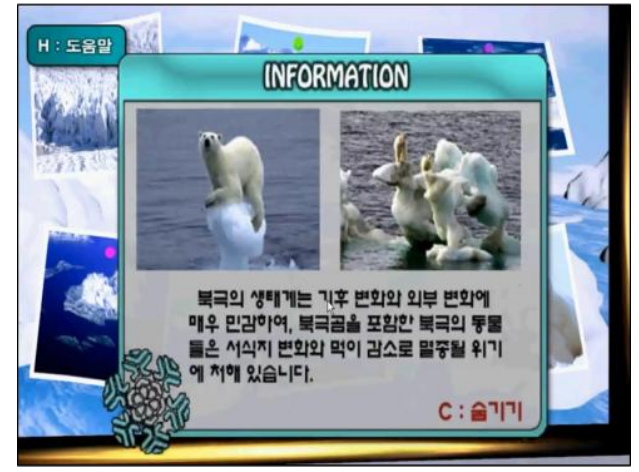

(e) Educational Content \#5

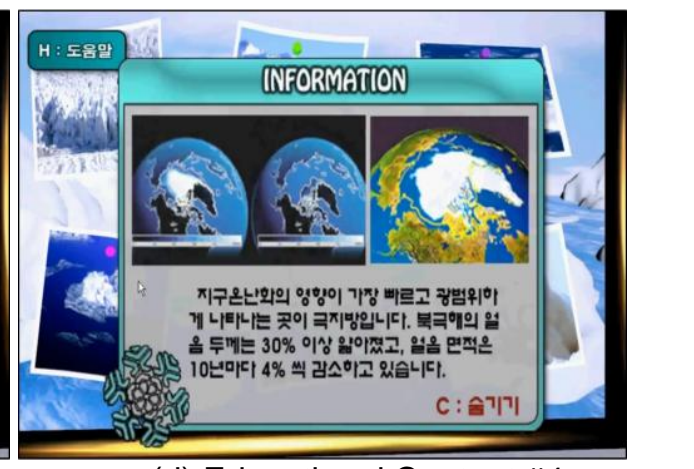

(d) Educational Content $* 4$

Figure 10. Educational contents of Learning Center in the Arctic

\subsection{Interaction}

This study deyeloped a cutting-edge virtual educational content that enables enjoyable and realistic interadtive experience based on engaging 3D virtual experience with a sense of reality the developed virtual educational content offers powerful user interactions, allowing participants to manipulate 3D objects with the mouse and keyboard. Based on the first-person avatar's perspective, participants can experience various places in the $3 \mathrm{D}$ environment and access diverse educational materials through multimedia information, including texts, sounds, and images. Moreover, participants' interactive experience was maximized through narrations. Figure 11 show the Interactive 3D technology of the Eco Center. 


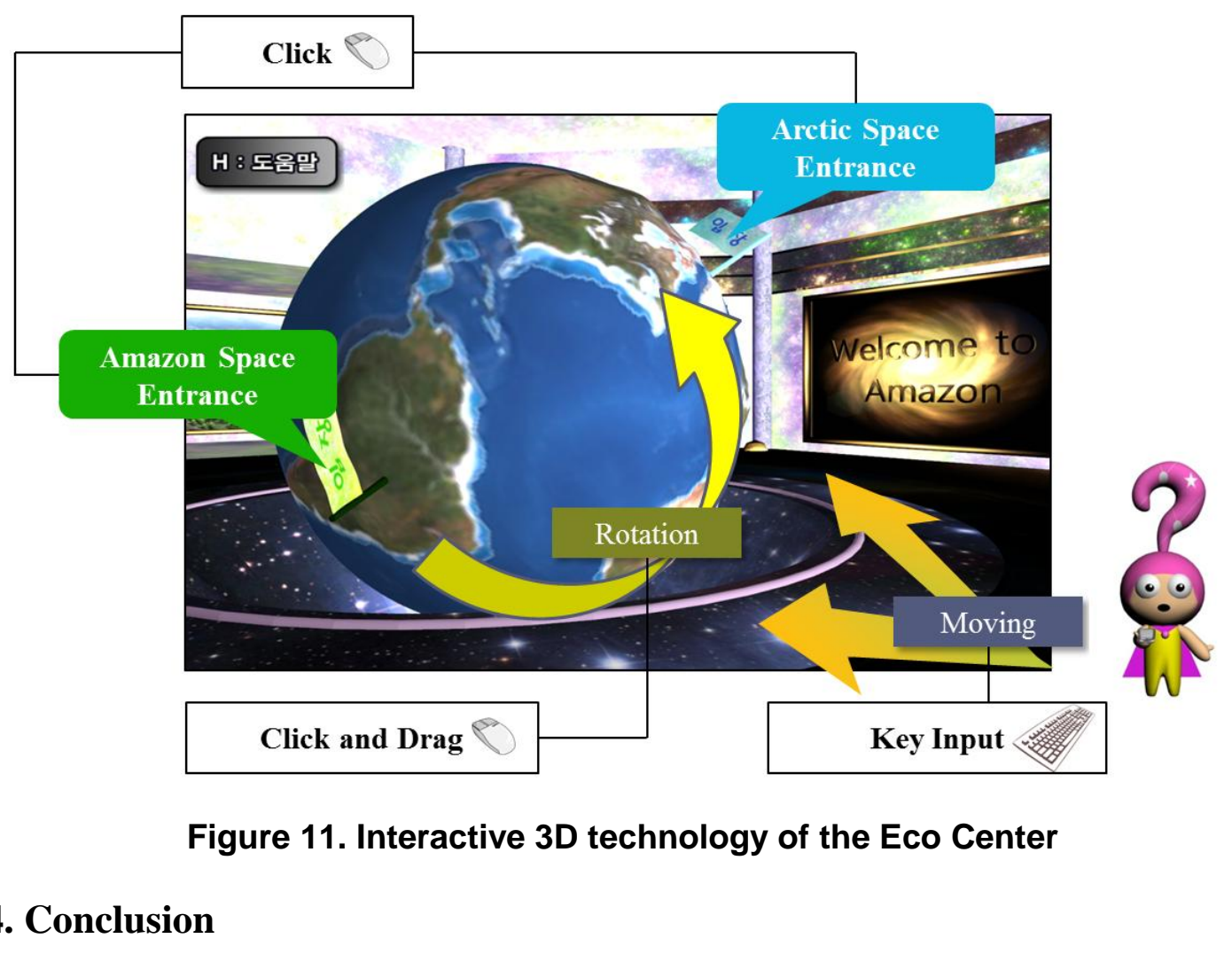

This paper proposed an intetactive Web 3D based virtual educational system using O3DWebGL. The proposed interactive Web $3 \mathrm{D}$ based virtual educational system is capable of providing flexible services without being confined to a specific platform. A virtual educational coptent was implemented with an educational goal of "saving the earth from global warming." The develoged virtual educational content of global warming consists of two subjects: Amazon, Arctic. The effectiveness of the proposed virtual educational system was verified based om implementation of user interaction. A storytelling approach was employed to maximize the interactive experience, and the 3D environment was implemented using O3D of WebGE to provide a user-oriented interface, as well as low-capacity, highquality data services. By applying a next-generation Web 3D technology, we were able to meet various demands from users and enhance the level of satisfaction.

\section{References}

[1] Y. Xia and K. Wang, "Integration Method of Multi-grid Evacuation Model in 3D Virtual Indoor Environment", International Journal of Multimedia and Ubiquitous Engineering, vol. 8, no. 3, (2013) May, pp. 255-264.

[2] J. O. Kim, M. Kim and K. H. Yoo, "Real-Time Hand Gesture-Based Interaction with Objects in 3D Virtual Environments", International Journal of Multimedia and Ubiquitous Engineering, vol. 8, no. 6, (2013), pp. 339-348.

[3] B. Chen and Z. Xu, "A Framework for Browser-based Multiplayer Online Games using WebGL and WebSocket", Proceedings of the International Conference on Multimedia Technology (ICMT), (2011) July, pp. 471-474.

[4] D. B. Chertoff, B. Goldiez and J. J. LaViola, "Virtual Experience Test: A virtual environment evaluation questionnaire”, IEEE Virtual Reality Conference (VR), (2010) March, pp. 103-110.

[5] K. Ponto, J. Kohlmann and M. Gleicher, "Effective Replays and Summarization of Virtual Experiences", IEEE Transactions on Visualization and Computer Graphics, vol. 18, no. 4, (2012), pp. 607-616. 
[6] M. Park and M. Gang, "Applying e-learning for Multicultural Healthcare Education”, International Journal of Multimedia and Ubiquitous Engineering, vol. 8, no. 6, (2013), pp. 367-376.

[7] M. M. Mobeen and L. Feng, "High-Performance Volume Rendering on the Ubiquitous WebGL Platform", Proceedings of the 14th International Conference on High Performance Computing and Communications, (2012) June, pp. 381-388.

[8] B. Steven, M. Jeanette, S. Dirk, P. Uta and P. Bernhard, "The LiverAnatomyExplorer: A WebGL-Based Surgical Teaching Tool”, IEEE Computer Graphics, vol. 33, no. 5, (2013), pp. 48-58.

[9] P. Danilicheva, S. Klimenko, Y. Baturin and A. Serebrov, "Education in Virtual Worlds: Virtual Storytelling", Proceedings of the International Conference on CyberWorlds, (2009), pp. 333-338.

[10] B. Chen, F. Huang and H. Lin, "Using Virtual world Technology to construct immersive 3D virtual university", Proceedings of the International Conference on Geoinformatics, (2009), pp. 1-5.

[11] M. Fominykh, E. Prasolova-Forland and M. Morozov, "From 3D Virtual Museum to 3D Collaborative Virtual Workshop", Proceedings of the IEEE International Conference on Advanced Learning Technologies, (2008), pp. 443-445.

[12] S. Klimenko, "Virtual Planetarium: Learning Astronomy in Virtual Reality", Proceedings of the World Conference on Educational Multimedia, Hypermedia and Telecommunications, (2004), pp. 2154-2157.

[13] S. Smith, S. Cutchin, A. Rockwood, A. Saad, N. G. Smith and T E. Levy, "Demo paper: Virtual and immersive experience presentation of Cultural Heritage Sites", Proceedings on the 18th International Conference on Virtual Systems and Multimedia (VSMM), (2012) September, pp. 645-648.

[14] http://www.khronos.org/webgl/.

[15] https://code.google.com/p/o3d/.

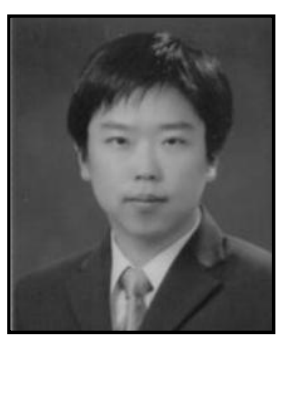

\section{Kwnag-Ho Seol}

He received the B.S. degree in internet-media engineering from Korea University of Technology and Education (KOREATECH), Korea, in 2007 and he M.S. degree, in Information media engineering, from Korea University of Tedlinology and Education (KOREATECH), Korea, in 2009. Since 2009 to now, he has been Ph.D. student in Humane Interaction Lab (HILab), Korea University of Technology and Education (KOREATECH), Cheonan, Korea. His current research interests include human-røbot interaction, and power system.

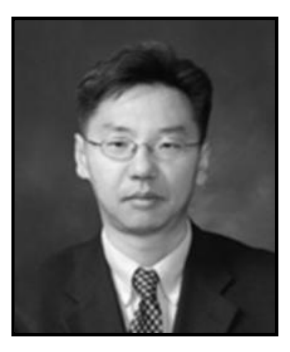

\section{Yoon Sang Kim}

He received the B.S., M.S., and Ph.D. degrees in Electrical Engineering from Sungkyunkwan University, Seoul, Korea, in 1993, 1995, and 1999, respectively. From 1999 to 2000 he was a member of the Postdoctoral Research Staff of Korea Institute of Science and Technology (KIST), Seoul, Korea. From 2000 to 2003 he was a Faculty Research Associate in the Department of Electrical Engineering, University of Washington, Seattle. From 2003 to 2005 he was a Member of the Senior Research Staff, Samsung Advanced Institute of Technology (SAIT), Suwon, Korea. Since 2005, he has been an Associate Professor at the School of Computer Science and Engineering, Korea University of Technology Education (KOREATECH), Cheonan, Korea. His current research interests include Virtual simulation, Power-IT technology, and Bio-informatics. Dr. Kim was awarded the Korea Science and Engineering Foundation (KOSEF) Overseas Postdoctoral Fellow in 2000. $\mathrm{He}$ is a member of IEEE, IEICE, ICASE, KIPS, and KIEE. 
International Journal of Multimedia and Ubiquitous Engineering Vol.9, No.4 (2014)

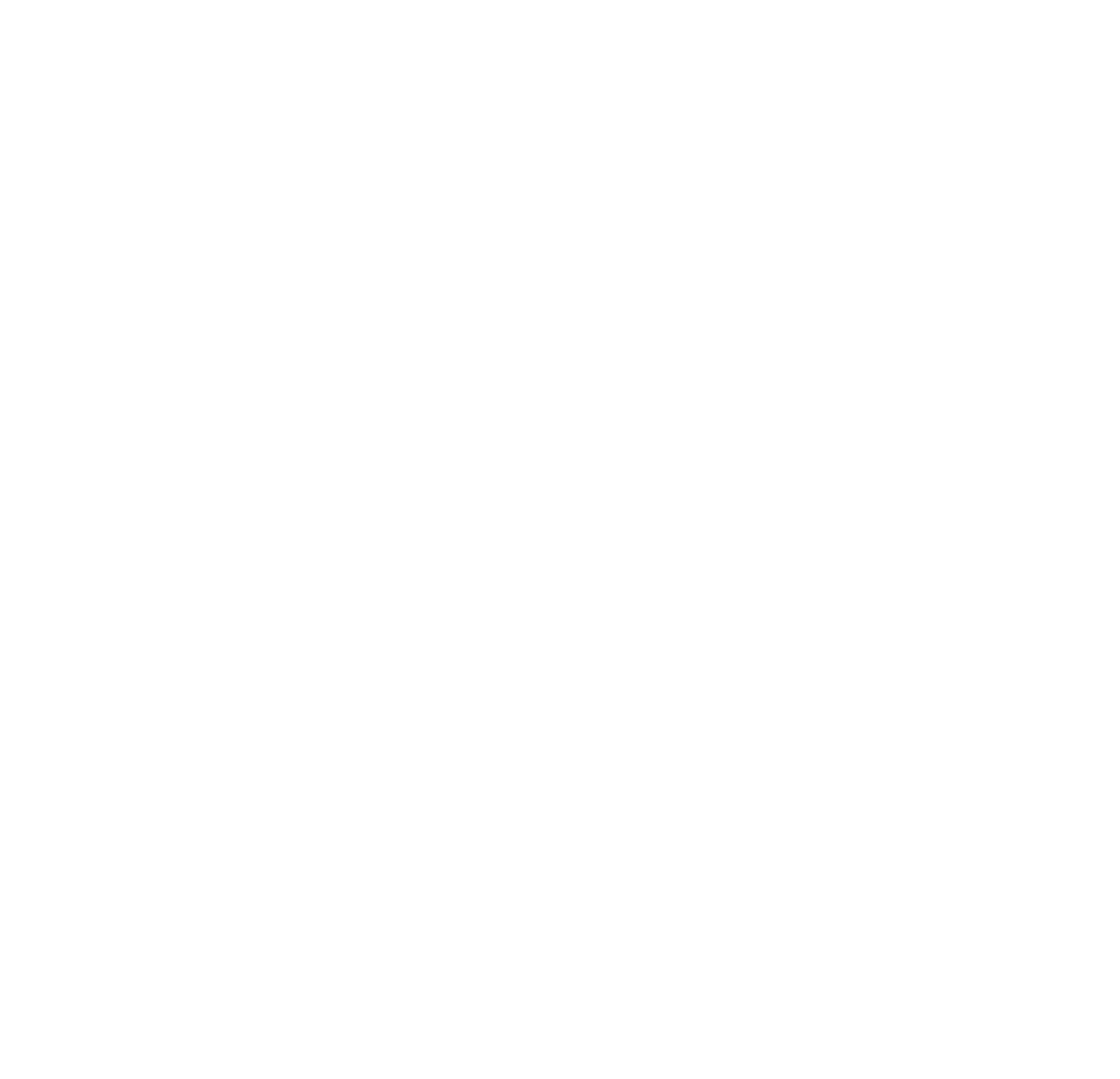

